\title{
Loneliness, Depression and Sociability in Old Age
}

\author{
Sisodia Devendra Singh $_{1}$
}

\begin{abstract}
:
The elderly population is large in general and growing due to advancement of health care education. These people are faced with numerous physical, psychological and social role changes that challenge their sense of self and capacity to live happily. Many people experience loneliness and depression in old age, either as a result of living alone or due to lack of close family ties and reduced connections with their culture of origin, which results in an inability to actively participate in the community activities. With advancing age, it is inevitable that people lose connection with their friendship networks and that they find it more difficult to initiate new friendships and to belong to new networks. The present study was conducted to investigate the relationships among depression, loneliness and sociability in elderly people. This study was carried out on 55 elderly people (both men and women). The tools used were Beck Depression Inventory, UCLA Loneliness Scale and Sociability Scale by Eysenck. Results revealed a significant relationship between depression and loneliness. Most of the elderly people were found to be average in the dimension of sociability and preferred remaining engaged in social interactions. The implications of the study are discussed in the article.
\end{abstract}

Keywords: Depression, Loneliness, Old age, Sociability

\section{INTRODUCTION:}

Aging is a series of processes that begin with life and continue throughout the life cycle. It represents the closing period in the lifespan, a time when the individual looks back on life, lives on past accomplishments and begins to finish off his life course. Adjusting to the changes that accompany old age requires that an individual is flexible and develops new coping skills to adapt to the changes that are common to this time in their lives (Warnick, 1995).

The diagnosis of disease should be complemented by assessment of discomfort associated with symptoms (e.g., pain), life threat, treatment consequences (e.g., side effects of medication), functional capacity and subjective health evaluations (Borchelt et al., 1999). Furthermore, Rowe \& Khan (1987) suggested that the health of subgroups of older adults be defined in terms of their status relative to age and cohort norms.

${ }^{1}$ Head,Department of Psychology,BN Girls College, MLS University,Udaipur

(C) 2015, S Singh; licensee IJIP. This is an Open Access Research distributed under the terms of the Creative Commons Attribution License (http://creativecommons.org/licenses/by/2.0), which permits unrestricted use, distribution, and reproduction in any Medium, provided the original work is properly cited. 


\section{Loneliness, Depression and Sociability in Old Age}

Depression or the occurrence of depressive symptomatology is a prominent condition amongst older people, with a significant impact on the well-being and quality of life. Many studies have demonstrated that the prevalence of depressive symptoms increases with age (Kennedy, 1996). Depressive symptoms not only have an important place as indicators of psychological well-being but are also recognized as significant predictors of functional health and longevity. Longitudinal studies demonstrate that increased depressive symptoms are significantly associated with increased difficulties with activities of daily living (Penninx et al., 1998). Community-based data indicate that older persons with major depressive disorders are at increased risk of mortality (Bruce, 1994). There are also studies that suggest that depressive disorders may be associated with a reduction in

These difficulties often emerge in older adulthood, increasing the likelihood of depression; yet depression is not a normal consequence of these problems. Studies have found that age isn't always significantly related to level of depression, and that the oldest of olds may even have better coping skills to deal with depression, making depressive symptoms more common but not as severe as in younger populations. Loneliness is a subjective, negative feeling related to the person's own experience of deficient social relations. The determinants of loneliness are most often defined on the basis of 2 causal models. The first model examines the external factors, which are absent in the social network, as the root of the loneliness; while the second explanatory model refers to the internal factors, such as personality and psychological factors.

Loneliness may lead to serious health-related consequences. It is one of the 3 main factors leading to depression (Green et al., 1992), and an important cause of suicide and suicide attempts. A study carried out by Hansson et al. (1987) revealed that loneliness was related to poor psychological adjustment, dissatisfaction with family and social relationships.

\section{OBJECTIVES OF THE STUDY}

- Examine the relationships among loneliness, depression and sociability in elderly persons

- Study gender differences with respect to sociability, loneliness and depression.

\section{HYPOTHESES}

- There will be a positive relationship between loneliness and depression in old age.

- There will be a negative relationship between sociability and loneliness in old age.

- There will be a negative relationship between sociability and depression in elderly persons.

- There will be gender differences with respect to the variables sociability, loneliness and depression in elderly persons. 


\section{MATERIALS AND METHODS}

\section{Sample}

The sample comprised of 60 elderly persons in the age group of 60-80 years. The subjects for the sample were selected among the older adults of Udaipur. These elderly persons were contacted personally, and the questionnaires were administered to them.

\section{Measures}

\section{The revised UCLA loneliness scale (Russell et al., 1980)}

The UCLA Loneliness Scale includes 10 negatively worded and 10 positively worded items that have the highest correlations with a set of questions that are explicitly related with loneliness. The revised version of the scale has high discriminative validity. The revised loneliness scale also has a high internal consistency, with a coefficient alpha of 0.94 .

\section{Beck depression inventory (Beck et al., 1961)}

The Beck Depression Inventory (BDI) is a 21-item self-report scale measuring supposed manifestations of depression. Sociability subscale of Eysenck personality profiler (Eysenck \& Eysenck, 1975)

Eysenck Personality Profiler (EPP V6) is a multidimensional modular personality inventory for 3 dimensions: Extroversion, emotionality (neuroticism) and adventurous ness (psychoticism). Each dimension has 7 subscales. The sociability subscale of extroversion used in this study consists of 20 questions. The response category is either 'yes' or 'no.' There are 10 positive items and 10 negative items.

\section{PROCEDURE}

Initially the participants were personally contacted and rapport was established with them. The participants completed the questionnaires given to them. Standard instructions were written on top of each questionnaire, and the participants were asked to rate themselves under the option they felt relevant to them. It was made clear to the participants that there were no right and wrong answers. If they had any difficulty, they were encouraged to ask questions. After finishing the entire set of questions, they were asked to return the questionnaires. The test administration took about 45 minutes. 


\section{RESULTS}

Table 1 shown above reveals that there are no significant gender differences in elderly men and women with respect to loneliness and depression. Elderly men, however, were found to be more sociable as compared to elderly women.

\begin{tabular}{|l|l|l|l|l|l|}
\hline Variables & Men & Women & t value \\
\hline & Mean & SD & Mean & SD & \\
\hline Loneliness & 47.43 & 7.54 & 45.75 & 0.73 & 0.47 \\
\hline Depression & 18.74 & 11.36 & 22.6 & 8.50 & 0.39 \\
\hline Sociability & 8.91 & 2.30 & 7.20 & 2.35 & 0.035 \\
\hline
\end{tabular}

Table 2 shows a significant positive correlation between depression and loneliness, which is significant at the 0.01 level, i.e., there is an increase in the level of depression with an increase in loneliness among elderly men and women. A negative, though insignificant, relationship was found between sociability and loneliness. No significant relationship was found between sociability and depression.

Correlations among loneliness, depression and sociability

\begin{tabular}{|l|l|l|l|}
\hline Variables & Loneliness & Depression & sociability \\
\hline Loneliness & 1.00 & & \\
\hline Depression & 0.528 & 1.00 & \\
\hline sociability & -0.010 & 0.032 & 1.00 \\
\hline
\end{tabular}

Table 3 reveals that in the male elderly persons, a significant positive correlation was found between depression and loneliness. Sociability and loneliness were negatively correlated, though not significantly.

Table 3 Correlations among loneliness, depression and sociability (men)

\begin{tabular}{|l|l|l|l|}
\hline Variables (Men) & Loneliness & Depression & sociability \\
\hline Loneliness & 1.00 & & \\
\hline Depression & 0.557 & 1.00 & \\
\hline sociability & -0.118 & 0.050 & 1.00 \\
\hline
\end{tabular}

Female elderly persons manifested a significant positive correlation between depression and loneliness, as can be seen in Table 4.

Table 4 Correlations among loneliness, depression and sociability (women)

\begin{tabular}{|l|l|l|l|}
\hline Variables (Women) & Loneliness & Depression & sociability \\
\hline Loneliness & 1.00 & & \\
\hline Depression & 0.602 & 1.00 & \\
\hline sociability & 0.165 & 0.265 & 1.00 \\
\hline
\end{tabular}




\section{Loneliness, Depression and Sociability in Old Age}

\section{DISCUSSION}

The health and well-being of older adults is affected by the level of social activity and the mood states. Researchers have reported the negative effects of loneliness on health in old age (Heikkinen et al., 1995). Loneliness, coupled with other physical and mental problems, gives rise to feelings of depression in the elderly persons. Gender differences have been reported in the prevalence of health problems in elderly persons (Arber \& Ginn, 1991). Results in Table 1 reveal that there are no significant gender differences in the elderly persons with respect to loneliness and depression, i.e., both the male and female elderly persons equally experience feelings of loneliness and depression. On the dimension of sociability, men were found to be more sociable as compared to their female counterparts.

This may have been due to the fact that all the elderly men belonged to the working group, i.e., they were employed in government jobs before retirement and were less hesitant in socializing as compared to their female counterparts who were housewives and were spending their lives at home and finding pleasures by engaging in daily chores. Having both the intellectual and social resources allows elderly men to continue to seek out new relationships.

Lack of significant gender differences on loneliness reflects the fact that since both the groups contained elderly married couples, with both partners being alive, the chances of their feeling lonely were low. Moreover, most of the couples were staying with their children and grandchildren, which did not allow them to stay lonely for long. Lack of significant gender differences on depression is contrary to the often held belief and research reports that elderly women are more prone to depression as compared to elderly men (Kessler et al., 1993). This result is not in line with what has been reported in literature. The findings of no significant gender differences with respect to depression may be attributed to the fact that all the women were nonworking ladies before they attained 60 years of age. Hence for them, the transition into old age was less associated with a change in life style associated with a break in ties with others or a sudden loss of power and status. The transition was very gradual, which prevented any abrupt change in mood states.

A positive correlation between loneliness and depression is in accordance with the results obtained in literature with regard to both male and female elderly persons (Green et al., 1992). No significant relationship between loneliness and sociability [Table 2] reveals that despite being sociable, they experienced increased feelings of loneliness. Possible explanation for this may be that feeling lonely not only depends on the number of connections one has with others but also whether or not one is satisfied with his life style. An expressed dissatisfaction with available relationships is a more powerful indicator of loneliness (Revenson, 1982).

Lack of significant relationship between depression and sociability [Table 2] confirms the fact that depression is multicausal, i.e., it arises due to a host of factors, like declining health, significant loss due to death of a spouse, lack of social support. Also most of the elderly persons 


\section{Loneliness, Depression and Sociability in Old Age}

had moderate connections with their friends and family members, and they participated in daily activities.

\section{On the basis of obtained findings, the following conclusions can be made:}

1. A significant positive correlation exists between loneliness and depression.

2. No significant relationship was found between loneliness and sociability; depression and sociability.

3. Men are found to be more sociable than women.

4. A significant correlation was found between loneliness and depression in both men and women.

5 .

\section{REFERENCES}

1. Arber S, Ginn J. Gender and later life. Sage, London: 1991.

2. Beck A. T, Ward C. H, Mendelson M, Mock J, Erbaugh J. An inventory for measuring depression. Archives of General Psychiatry. 1961;4:561-571.

3. Borchelt M, Gilberg R, Horgas A. I, Geiselmann B. On the significance of morbidity and disability in old age. In: Baltes P.B, Mayer K.U, editors. The Berlin Aging Study: Aging from 70 to 100. New York: Cambridge University Press; 1999. pp. 403-429.

4. Bruce M. L, Seeman T, Merrill S. S, Blazer D. G. The impact of depressive symptomatology on physical disability: MacArthur Studies of successful aging. American Journal of Public Health. 1994;84:1796-1799.

5. Charles S. T, Reynolds C. A, Gatz M. Age-related differences and change in positive and negative affect over 23 years. Journal of Personality and Social Psychology. 2001;80:136-151.

6. Costa P. T, Jr, McCrea R. R. Influence of extra version and neuroticism on subjective well-being: Happy and Unhappy people. Journal of Personality and Social Psychology. 1980;38:668-678

7. Diener E, Suh E. M, Lucas R. E, Smith H. L. Subjective well-being: Three decades of progress. Psychological Bulletin. 1999;125:276-302.

8. Eysenck H. J, Eysenck S. B. G. Manual of the Eysenck Personality Questionnaire (adult \& junior) London: Hodder \& Stoughton; 1975.

9. George L. K. Social factors and illness. In: Binstock R.H, George L.K, editors. Handbook of aging and the social sciences. 4thed. San Diego, CA: Academic Press; 1996. pp. 229-253.

10. Green B. H, Copeland J. R, Dewey M. E, Shamra V, Saunders P. A, Davidson I. A, Sullivan C, McWilliam C. Risk factors for depression in elderly people: A prospective study. Acta Psychiatr Scand. 1992;86(3):213-7.

11. Hansson R. O, Carpenter B. N. Relationships in Old Age: Coping with the challenge of transition. New York, NY: Guilford Press; 1994. 


\section{Loneliness, Depression and Sociability in Old Age}

12. Hansson R. O, Jones W. H, Carpenter B. N, Remondet J. H. International Journal of Human Development. 1986-1987;27(1):41-53.

13. Heikkinen R, Berg S, Avland K. Depressive symptoms in late life. Journal of Cross Cultural Gerontology. 1995;10:315-330.

14. Kennedy G.J. The epidemiology of late-life depression. In: Kennedy G. J, editor. Suicide and depression in late life: Critical issues in treatment, research and public policy. New York: John Wiley and Sons; 1996. pp. 23-37.

15. Max L. S, David J. V, Jacobijn G, Aartjan T. F, Ross van der Mast \& Rudi G. J. Is depression in old age fatal only when people feel lonely? American Journal Psychiatry. 2005;162:178-180.

16. Mullins L. C, Johnson D. P, Anderson L. Danish Medical Bulletin. Special Supplement Series, No. 6, October. 1988:26-29.

17. Penninx B. W, Guralnik J. M, Ferrucci L, Simonsick E. M, Deeg D. J, Wallace R. B. Depressive symptoms and physical decline in community-dwelling older persons. Journal of the American Medical Association. 1998;127:1720-1726.

18. Posner R. Aging and Old Age. USA: University of Chicago Press; 1995.

19. Revenson T. A. Predictable loneliness of Old Age: Dispelling the Myth. Paper presented at the Annual Convention of the American Psychological Association (90th Washington, DC, August 23-27, 1982) 1982

20. Rowe J. W, Khan R. L. Human aging: Usual and successful. Science. 1987;237:143149.

21. Russell D, Peplau L. A, Cutrona C. E. The revised UCLA Loneliness Scale: Concurrent \& discriminant validity evidence. Journal of Personality \& Social Psychology. 1980;39:472-480.

22. Smith J, Fleeson W, Geiselmann B, Settersten R. A, Jr, Kunzmann U. Sources of wellbeing in very old age. In: Baltes P.B, Mayer K.U, editors. The Berlin Aging Study: Aging from 70 to 100. New York: Cambridge University Press; 1999. pp. 450-471.

23. Speck C. E, Kukull W. A, Brenner D. E, Bowen J. D, McCormick W. C, Teri W. C, Pfanschmidt M. L, Thompson J. D, Larson E. B. History of depression as a risk factor for Alzheimer's disease. Epidemiology. 1995;6:366-369.

24. Warnick J. Listening with different ears: Counseling people over sixty. Ft. Bragg CA: QED Press; 1995.

25. Wulsin L. R, Vaillant G. E, Wells V. E. A systematic review of the mortality of depression. Psychosomatic Medicine. 1999;161:6-17. 